Original paper

\title{
MaxiCalc: A tool for online dosimetric evaluation of source-tracking based treatment verification in HDR brachytherapy
}

\author{
Maximilian D. Hanlon ${ }^{\text {a,b, }}$, Ryan L. Smith ${ }^{\mathrm{a}, \mathrm{b}}$, Rick D. Franich ${ }^{\mathrm{a}}$ \\ ${ }^{\text {a }}$ School of Science, RMIT University, Melbourne, Australia \\ ${ }^{\mathrm{b}}$ Alfred Health Radiation Oncology, The Alfred, Melbourne, Australia
}

\section{A R T I C L E I N F O}

\section{Keywords:}

HDR brachytherapy

Treatment verification

Dose calculation

Adaptive brachytherapy

DVH indices

Source tracking

\begin{abstract}
A B S T R A C T
Purpose: Source tracking is becoming a more widely used approach in HDR brachytherapy treatment verification. While it provides a sensitive method to detect deviations from the treatment plan during delivery, it does not show the clinical significance of any detected changes. By incorporating a tool that calculates volumetric doses and DVH indices from measurements, source tracking systems can be expanded to assess dosimetric significance of any deviations from the plan.

Methods: The source tracking dose calculation tool, MaxiCalc, was developed in MATLAB. Validation was performed by comparing doses and DVH indices calculated in MaxiCalc to those calculated by the clinical TPS, for several test plans and 10 clinical plans. Clinical implementation was demonstrated by calculating volumetric doses from a clinical source tracking event.

Results: MaxiCalc showed excellent agreement with the clinical TPS for point and volumetric doses (mean difference $<0.01 \%$ and $0.1 \%$ respectively). MaxiCalc calculates dosimetrically equivalent plans to the TPS with agreement $<0.3 \%$ for all DVH indices except PTV V200\%. Small differences seen for the clinical source tracking event were consistent with the known tracking uncertainties enabling them to be quantified for clinical decision making. Calculations are fast, enabling real-time use.

Conclusions: MaxiCalc is an independent tool that calculates doses and DVH indices from dwells measured with any clinical HDR brachytherapy source tracking system. This extends the capabilities of source tracking systems from determining discrepancies in positions or times during delivery to assessing the dosimetric impact of any detected deviations, allowing for more comprehensive treatment verification and evaluation.
\end{abstract}

\section{Introduction}

Since our earliest work in the development of a HDR brachytherapy verification system based on a flat panel detector, [1] it has been clear that source tracking provides a wealth of additional information that is not necessarily readily available from in vivo dosimetry (IVD) alone. Our focus evolved from demonstrating a non-invasive dosimetric verification system [2] to improvements in source tracking accuracy coupled with improved tools for comparison to the intended plan. [3] The richness of information from source tracking extends the capabilities of a treatment verification system from the primary aims of error detection and patient safety, to monitoring smaller changes - for example due to implant movements between planning and treatment delivery. While such changes are known to occur, there are few practical strategies for assessing their significance or potential impact on patient outcomes.
Several groups with well-developed IVD systems have recognised the value of source tracking, either by measuring the individual 3D source positions or by measuring implant movements relative to the in vivo dosimeter. [4-8] Other groups have performed source tracking via other methods of directly measuring the source position with detectors including fluoroscopy, pinhole cameras, magnetic resonance, or 2D arrays. [3,9-14] Some of the source tracking approaches have been demonstrated clinically, $[3,7,9,15]$ or in principle $[5,6,8,10-13,16]$.

The source tracking approach typically determines the spatial position of the radioactive source during treatment delivery and compares this with expected position derived from the treatment plan. If the verification system is capable of capturing the time the source dwells at each position, it follows that the volumetric dose that was delivered is able to be reconstructed using the TG-43U1 formalism. [17] If the anatomical relationship is known relative to the measured dwell

\footnotetext{
* Corresponding author at: School of Science, RMIT University, Melbourne, Australia.

E-mail address: MaxDHanlon@gmail.com (M.D. Hanlon).
} 
positions and times, then a dosimetric analysis can be performed potentially comparing the expected dose volume histogram (DVH) indices with the planned DVH indices. A recent review into HDR brachytherapy in vivo dosimetry and treatment verification [18] highlighted that the most clinically relevant metrics involve DVH calculations. Combining the novel software system described in this work with a clinical source tracking system capable of measuring the dwell positions and times during treatment achieves a more comprehensive treatment verification system where the delivered dose can be calculated and assessed.

In previous work, we have shown that source tracking for treatment verification is a viable solution in the clinical environment, and our system has been in routine use for every fraction across every patient since 2015. [3] The use of a flat panel detector (FPD) for source tracking enables pre-treatment imaging to be performed with the same system. In addition to implant evaluation, this provides the relationship between measured dwell positions and the patient's anatomy. The entire process is non-invasive, reproducible, and is readily integrated into the current clinical workflows. The current clinical challenge is to sensibly use the large amount of data the system produces in order to make a dosimetric evaluation of the treatment.

The progression of brachytherapy treatment verification in the clinic has exceeded the commercial development of useful tools to assist and enable this as a routine process. In particular, no viable tools exist that can reconstruct a volumetric dose grid from a collection of measured source dwells (i.e., dwell positions and dwell times) acquired during a source tracking verification event. While TPSs calculate the planned volumetric dose distribution, all calculations are limited to dwell positions that reside in channel paths as defined in pre-treatment planning imaging. To accommodate the nature of the measured source dwells, the software tool is required to reconstruct dose for any arbitrary point in space that represents the patient treatment region. This allows for catheter movements that are often responsible for small discrepancies between planned and measured dwell positions to be correctly accounted for. To allow for dosimetric assessment, the tool should also calculate DVH indices. Furthermore, a tool that can calculate a reconstructed dose in real time (i.e., during the treatment delivery) provides the clinical staff with feedback on dynamic dose indices information, that could be compared with planned dose indices, and be potentially used as a treatment interrupt flag if clinically relevant deviations are observed. In addition to being able to provide real time dosimetric index calculations, the same system allows for more representative dose indices to be calculated for every delivered plan, improving the recording of delivered dose indices. By using this more representative dose instead of the planned dose, adaptive planning strategies can be realised, and the quality of radiobiological and outcome studies is improved.

A recent study into in vivo source tracking error thresholds found that there was significant patient-to-patient deviation of some DVH indices, such as the Urethra D1cc. [19] That study performed the dosimetric calculations in the clinical TPS, which is a time consuming method that is not feasible as a routine, patient specific approach. By automating this process, patient and plan specific error thresholds can be determined that take into account the impact that any detected spatial deviations of the treatment delivery have on the dosimetry.

The aim of the present work is to establish a software tool that can meet the needs of the clinic for source tracking treatment verification in HDR brachytherapy. MaxiCalc is designed to perform dose calculations that are not readily possible with clinical TPSs. Namely, the dose distribution from dwell positions measured during treatment delivery which are not necessarily identical to those in the plan, and may not even align within the catheter paths defined in the plan. This dose calculation cannot be done (at least not without significant manual work) in the TPS since dwell positions cannot be defined outside of the defined catheters. We discuss the implementation of such a tool and detail a method to test and validate this independent dose analysis tool for the interpretation of source-tracking data in terms of clinical significance. The ability of the tool to calculate volumetric doses and DVH indices is shown, along with the ability to generate this data for a clinically delivered patient plan where the source was tracked throughout treatment delivery.

\section{Method}

MaxiCalc imports the measured dwell positions and times, the treatment plan data, performs dose calculations, and analyses and compares any differences between the delivered and planned doses in terms of dose differences and DVH index differences. The validation of MaxiCalc is performed in three stages: determining the agreement of the dose calculation engine with clinically used TPSs, validating the implementation of the DVH index calculations, and combining these to show that MaxiCalc generates doses with the same DVH indices as the clinical TPS for identical dwells.

MaxiCalc was developed in MATLAB v2017b (MathWorks, Massachusetts, USA). The attributes for the source type are loaded dynamically, which allows for any brachytherapy source with published dosimetric data to be used. The dose calculations used to validate MaxiCalc have been performed using the microSelectron mHDR v2 source (Elekta AB, Stockholm, Sweden) as this is the clinical source and source model used at the centre and at the time where the software was developed. The source data for this source is taken from work by Daskalov, Löffler, and Williamson. [20] Two sets of look up tables (LUTs) for the anisotropy and radial dose functions are used, one taken from the paper verbatim, and one with extrapolated and interpolated values that matches the LUTs used in the TPS. All TPS calculations are performed in Oncentra Brachy (OCB) v4.6 (Elekta AB, Stockholm, Sweden). In this manuscript, this analysis is shown for HDR brachytherapy prostate treatments, as this is the most common HDR brachytherapy treatment at the clinic where the research is based.

\subsection{Dose calculation}

MaxiCalc was coded with a universal approach to allow for different types of dose calculations. It can calculate point doses using the TG43U1 formalism to explicitly calculate dose at each point using published data, or it can calculate volumetric doses of specified dose grid size and resolution. Both approaches allow for the calculation of dose from one or many measured source dwell positions.

\subsubsection{Point dose module}

MaxiCalc calculates point doses explicitly using the TG-43 general 2D formalism. To allow for this calculation, the source specific dosimetric data (dose rate constant, LUTs for the anisotropy function and radial dose function) are loaded at runtime. The radius (r) and angle from the source $(\theta)$ are calculated as per the definition in TG-43U1 and used to calculate the geometry function values that correspond to that point. The same values $(r, \theta)$ are used to interpolate the corresponding values from the radial dose function and anisotropy function LUTs using a linear and bilinear interpolation respectively.

The implementation of the TG-43U1 algorithm in MaxiCalc was validated by calculating the dose rate (dose per unit air-kerma strength) at the same set of points $(\mathrm{N}=27)$ in the along and away table used to validate the planning system. [21] The distance to these points ranges from 1 to $6.4 \mathrm{~cm}$ away from the source. The dose rate values are calculated in MaxiCalc using two sets of LUTs (OCB and literature) and are then compared to values calculated by OCB. The agreement is assessed in terms of local absolute dose difference.

\subsubsection{Volumetric dose module}

MaxiCalc calculates volumetric doses with two methods: (i) TG43: an explicit TG-43 based calculation of dose at each voxel from each source, or (ii) 2DAA: interpolating the dose at each reference position from a 
pre-calculated 2D Along-and-Away table. The TG-43 approach is used as it provides a calculation with fewer interpolations and therefore a more accurate representation of the TG-43U1 formalism. The along-and-away approach was used to match that of the TPS to allow for direct comparison.

The TG43 method, (i), involves stepping through each measured dwell position and determining the distance $(r)$ and angle $(\theta)$ of each point in the calculation grid from the dwell position. The angle of the source is considered if it is known, which may not be the case depending on what the inputs to MaxiCalc are (e.g., a source tracking treatment verification system without the accuracy to determine source orientation). The dose for each dwell position is then calculated in the same manner as in Section 2.1.1, and the total volumetric dose is the sum of the contributions from each dwell position measured during a verification event.

The 2DAA method, (ii), also steps through each measured dwell position. The distance of each voxel along and away relative to the dwell position (distance along the source axis and the distance away from the central transverse axis) is used to calculate dose from a precalculated dose table with a 2D bilinear interpolation. The precalculated dose table is a source-specific along and away table with values at $1 \mathrm{~mm}$ resolution in both the along and away directions. This method was chosen as it is the method used in the TPS and is therefore expected to provide better agreement.

The TG-43 method is computationally expensive as the TG-43U1 approach requires calculating the geometry function, the distance, and the angle for each reference position and dwell position, and then forming interpolations for the contribution due to radial dose function and anisotropy function. The advantage of using the 2DAA approach is that only the along and away distances need to be calculated, and then only one interpolation for each reference position and dwell position needs to be performed. The 2DAA approach is expected to show better agreement with the TPS, but it is expected to be less representative of the TG-43U1 formalism due to the increased number of interpolations used. While the TG-43U1 protocol is considered the standard when calculating dose in brachytherapy, modern TPSs do not calculate this explicitly but rather calculate the dose by interpolating through pre-calculated LUTs. Because of this, the dose calculated by the TPS is taken to be the reference dose for all comparisons and validations.

To validate these volumetric dose calculation methods, a simple plan was made for a phantom with catheters (Proguide 6F, Elekta, Stock-

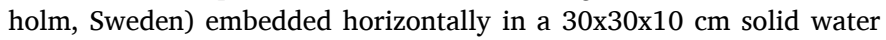
phantom, shown in Fig. 1. The plan had 117 active dwell positions across 9 catheters, with 13 dwells per catheter. Dwells were $2.5 \mathrm{~mm}$ apart, spanning a total distance of $30 \mathrm{~mm}$, and had dwell times of 1,2 , or $3 \mathrm{~s}$ assigned randomly. MaxiCalc imported the required plan data (dwell position data, source air kerma strength, and prescription dose) from the DICOM-RT plan file to recalculate the planned dose. To allow for like-

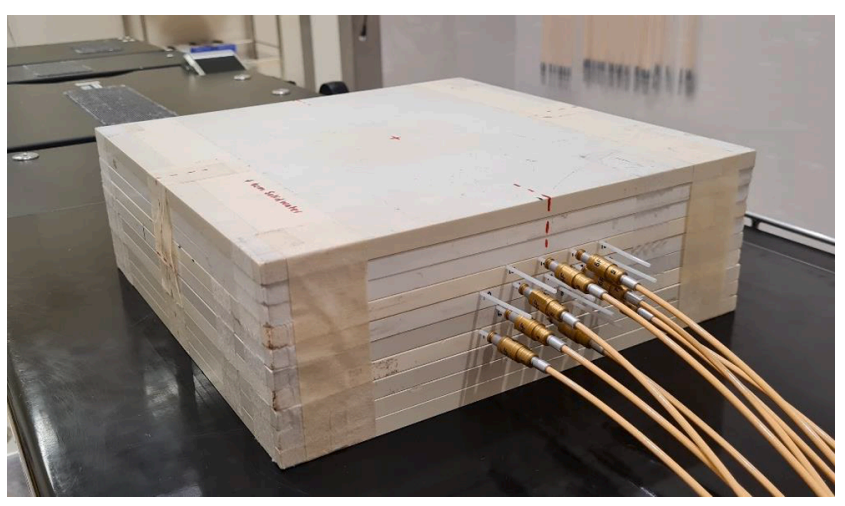

Fig. 1. The solid water phantom with transfer tubes connecting 9 embedded catheters to the remote afterloader. for-like comparison, the OCB-calculated dose is imported from the DICOM-RT dose file. For these comparisons, both OCB and MaxiCalc calculated with dose grids of $1 \mathrm{~mm}^{3}$ cubic voxels, and local dose differences were assessed across the entire volume.

To test the timing and efficiency of MaxiCalc, the dose for a clinical plan was calculated with both the 2DAA and TG43 methods. The treatment plan had 420 dwell positions, and the calculation grid was $124 \times 104 \times 123 \mathrm{~mm}$ with a resolution of $1 \mathrm{~mm}^{3}$ voxels, for a total of over 1.5 million voxels. The calculations were run on a laptop computer with an Intel Core i7-1165G7 processor and 16 GB of RAM.

\subsection{DVH calculations}

To provide clinically useful dose indices from clinical source tracking, MaxiCalc has the capacity to calculate DVHs. The anatomical structure information is extracted from the DICOM-RT files exported from OCB using code adapted from BrachyGUI. [22] The structure volumes used to calculate DVHs are represented by a binary mask of the same dimensions as the dose grid for each structure.

\subsubsection{Validation of DVH index calculations}

Validation of the structure definitions and DVH calculations in MaxiCalc was performed by comparing the DVH indices calculated in MaxiCalc for a dose calculated in OCB to the DVH indices calculated in OCB for the same plan. Using the exported OCB dose distribution ensured that the DVH index calculations were independent of any influence of dose calculation method.

The DVH indices validated were the same indices used clinically at Alfred Health Radiation Oncology (AHRO) for HDR prostate treatments, namely:

- PTV D90\% (the percentage of prescription dose delivered to $90 \%$ of the volume of the PTV)

- PTV V100\%, V150\%, and V200\% (the volume of the PTV that receives $100 \%, 150 \%$, and $200 \%$ of the prescribed dose respectively)

- Rectal wall D2cc (the maximum dose received by $2 \mathrm{~cm}^{3}$ of the rectal wall)

- Urethra D10\% (the percentage of the prescription dose delivered to $10 \%$ of the volume of the urethra)

- Bladder V84\% (The volume of the bladder that receives $84 \%$ of the prescription dose)

\subsubsection{DVH index calculations from MaxiCalc dose grids}

While Section 2.2.1 validates the calculation of DVH indices in MaxiCalc, this section validates that the volumetric doses calculated in MaxiCalc generate equivalent DVH indices to the volumetric doses calculated by OCB. This is shown in two ways. Firstly, a cumulative DVH was calculated in MaxiCalc for a single plan for a qualitative assessment of agreement. Secondly, 10 clinical dose grids were exported from the TPS, imported into MaxiCalc, and the DVH indices were calculated in the same manner as Section 2.2.1 and used as reference DVH indices. MaxiCalc was then used to calculate the volumetric doses for the 10 plans in the same manner as Section 2.1.2 with both methods (TG43 and 2DAA) and both sets of LUTs (OCB and literature) and then calculate the resultant DVH indices. The mean difference between the DVH indices for the dose grids calculated with MaxiCalc and the reference DVH indices were then compared.

\subsection{Dose calculations from tracked dwell positions}

The dose was calculated in MaxiCalc for a clinically delivered plan where the source was tracked during delivery, providing a set of measured dwell positions and times. The source tracking was performed in the same manner as previously published work. [3] The measured source positions were transformed from the measurement space (the frame of reference of the source tracking system) into the TPS space (the 
frame of reference of the clinical dwell positions in the TPS) for dose calculations using a registration derived from gold fiducial makers implanted in the prostate. The dwell positions were then imported into MaxiCalc. As the source tracking system currently provides a lowresolution measurement of dwell times, the planned dwell times were used in the dose calculation, for all dwell times within the time measurement uncertainty. The dose calculations were performed with the 2DAA method and the OCB LUTs to allow direct comparison with the TPS-calculated DVH indices.

\section{Results}

\subsection{Dose calculation}

\subsubsection{Point dose module}

The absolute local differences in dose calculated for the 27 validation points between MaxiCalc and OCB is shown in Table 1 for both the literature LUTs and the interpolated and extrapolated LUTs used in OCB. [21] The 27 dose points span a range from 1 to $6.4 \mathrm{~cm}$ away from the source. All non-zero differences can be attributed to OCB data being truncated at 4 decimal places. The full data comparison is reproduced in the Supplementary Data 1.

\subsubsection{Volumetric dose module}

Table 2 shows local dose differences between MaxiCalc and OCB for the phantom volumetric plan consisting of 117 dwell positions. The mean signed and absolute dose differences are shown along with the standard deviation of the differences, and the percentage of voxels with differences below $0.5 \%$ and $2 \%$, where $2 \%$ is threshold agreement recommended in TG43U1. Both the full TG43 method and the 2D along and away (2DAA) methods are shown for both sets of LUTs.

As Table 2 includes dose comparisons for a large proportion of relatively low-dose voxels, Table 3 shows the local dose differences for the clinically relevant sub-volume receiving $50-200 \%$ of the prescription dose, and correlates to regions within approximately 0.2 to $2 \mathrm{~mm}$ (200\%) and 5.5 to $15 \mathrm{~mm}$ (50\%) of active dwell positions, depending on dwell times. This range was chosen as it is the order of clinically significant distances. This represents $<1 \%$ of the dose calculation volume and demonstrates that 2DAA has much better agreement with the TPS, emphasizing that matching the dose calculation algorithm is more important than matching the LUTs used.

The calculations for the clinical plan consisting of 420 dwell positions took $97 \mathrm{~s}$ for the full TG43 approach, and $34 \mathrm{~s}$ for the 2DAA interpolation approach, for a time of $0.23 \mathrm{~s}$ and $0.08 \mathrm{~s}$ per dwell position respectively.

\subsection{DVH calculations}

\subsubsection{Validation of DVH index calculations}

Table 4 shows the DVH indices calculated in OCB and in MaxiCalc for a clinical plan where the dose was generated in OCB. Good agreement can be seen between the two programs.

\section{Table 1}

Local dose differences between MaxiCalc (two LUTs) and OCB for the same 27 points used to validate Oncentra Brachytherapy (OCB) v4.6 as per the OCB documentation. The TG43 implementation of MaxiCalc using the two different LUTs is compared to the TPS. The data is showing the mean and maximum unsigned (i.e., absolute) differences, as well as the number of points with differences $>0.1 \%$ and $0.2 \%$.

\begin{tabular}{lllll}
\hline LUT & Mean (abs) [\%] & Max (abs) [\%] & $\#>0.1 \%$ & $\#>0.2 \%$ \\
\hline OCB & 0.00 & 0.01 & $0 / 27$ & $0 / 27$ \\
Literature & 0.07 & 0.30 & $9 / 27$ & $2 / 27$ \\
\hline
\end{tabular}

Table 2

Local dose differences between MaxiCalc generated dose grids and OCB exported dose grids for identical plans and dose grid size, location, and resolution. All differences represent the percentage local difference, where a value $>0$ means that the OCB generated dose is lower than the MaxiCalc generated dose. The calculation methods are the TG43 method and the $2 \mathrm{D}$ along and away method (2DAA).

\begin{tabular}{|c|c|c|c|c|c|}
\hline LUT source & $\begin{array}{l}\text { Calculation } \\
\text { method }\end{array}$ & $\begin{array}{l}\text { Mean } \\
\text { (s.d.) }\end{array}$ & $\begin{array}{l}\text { Absolute } \\
\text { mean (s.d.) }\end{array}$ & $\begin{array}{l}\text { Voxels < } \\
0.5 \%\end{array}$ & $\begin{array}{l}\text { Voxels } \\
<2 \%\end{array}$ \\
\hline & & [\%] & [\%] & [\%] & [\%] \\
\hline \multirow[t]{2}{*}{$\begin{array}{l}\text { Literature } \\
\text { LUTs }\end{array}$} & Full TG43 & $\begin{array}{l}-0.08 \\
(0.7)\end{array}$ & $0.13(0.7)$ & 97.6 & 99.7 \\
\hline & 2DAA & $\begin{array}{l}-0.05 \\
(0.7)\end{array}$ & $0.09(0.7)$ & 98.5 & 99.9 \\
\hline \multirow[t]{2}{*}{ OCB LUTs } & Full TG43 & $\begin{array}{l}-0.01 \\
(0.6)\end{array}$ & $0.07(0.6)$ & 97.5 & 99.7 \\
\hline & 2DAA & $\begin{array}{l}0.02 \\
(0.7)\end{array}$ & $0.05(0.7)$ & 98.3 & 99.9 \\
\hline
\end{tabular}

Table 3

Local dose differences for doses between $50 \%$ and $200 \%$ of the prescription dose for the same plans as Table 2 .

\begin{tabular}{|c|c|c|c|c|}
\hline & & Mean (s.d.) & Voxels $<0.5 \%$ & Voxels $<2 \%$ \\
\hline & & [\%] & [\%] & [\%] \\
\hline \multirow[t]{2}{*}{ Literature LUTs } & Full TG43 & $1.5(2.4)$ & 31.0 & 80.6 \\
\hline & 2DAA & $0.2(1.0)$ & 97.2 & 99.6 \\
\hline \multirow[t]{2}{*}{ OCB LUTs } & Full TG43 & $1.4(2.4)$ & 37.2 & 81.9 \\
\hline & 2DAA & $0.1(0.9)$ & 98.2 & 99.9 \\
\hline
\end{tabular}

Table 4

The calculated dose indices from Oncentra Brachy V4.6 and MaxiCalc for a plan calculated in Oncentra Brachy. For MaxiCalc, the DVH indices were calculated using the DICOM-RT dose grid exported from OCB.

\begin{tabular}{|c|c|c|c|c|}
\hline & \multirow[b]{2}{*}{ DVH index } & \multicolumn{2}{|c|}{$\begin{array}{l}\text { DVH index calculation } \\
\text { software }\end{array}$} & \multirow[b]{2}{*}{ Difference $[\%]$} \\
\hline & & OCB & MaxiCalc & \\
\hline \multirow[t]{4}{*}{ Prostate (PTV) } & D90\% [\%] & 102.0 & 100.9 & -1.1 \\
\hline & V100\% [\%] & 91.7 & 90.6 & -1.2 \\
\hline & V150\% [\%] & 18.1 & 18.1 & 0.2 \\
\hline & V200\% [\%] & 7.6 & 7.7 & 0.9 \\
\hline Rectal wall & D2cc [\%] & 65.1 & 66.1 & 1.5 \\
\hline Bladder & $\mathrm{V} 84 \%\left[\mathrm{~cm}^{3}\right]$ & 2.5 & 2.6 & 2.8 \\
\hline Urethra & D10\% [\%] & 107.1 & 106.6 & -0.5 \\
\hline
\end{tabular}

\subsubsection{DVH index calculations from MaxiCalc dose grids}

A cumulative DVH is presented in Fig. 2 for one of the clinical plans showing the doses calculated in both OCB and MaxiCalc. The MaxiCalc dose was calculated with the 2DAA method and the LUTs exported from OCB. Very good agreement is seen for all four structures.

To show the DVH index agreement with OCB over multiple plans, a distribution of the differences in the primary target coverage metric, PTV D90\%, is shown as a box and whisker plot in Fig. 3. All DVH indices were calculated in MaxiCalc for the 4 algorithm variants, i.e., 2 calculation methods and the 2 sets of LUTs. The DVH indices for each combination of LUT and method were compared. The PTV D90\% is shown as a representative index, and all other indices except for the PTV V200\% showed similar or better agreement. For completeness, the 6 remaining clinical indices used are provided in the Supplementary Material 2.

\subsection{Dose calculations from tracked dwell positions}

The DVH indices for a clinically delivered plan in which source tracking was performed are shown in Table 5. Minimal deviations from the plan are seen for the measured DVH indices. 


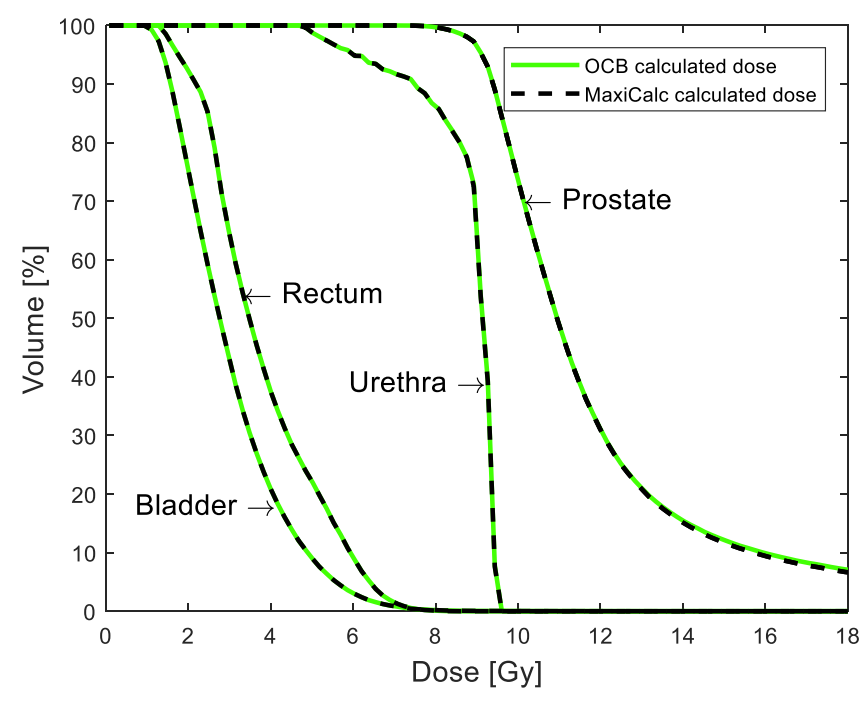

Fig. 2. A cumulative DVH for a clinical plan comparing the dose indices for the plan calculated in and exported from OCB (OCB calculated dose - green solid line) and the dose calculated with MaxiCalc (MaxiCalc calculated dose - black dashed line). The prescription dose is $8.5 \mathrm{~Gy}$, and all dose index calculations were performed in MaxiCalc with the OCB LUTs and the 2DAA approach due to it having the best agreement. (For interpretation of the references to colour in this figure legend, the reader is referred to the web version of this article.)

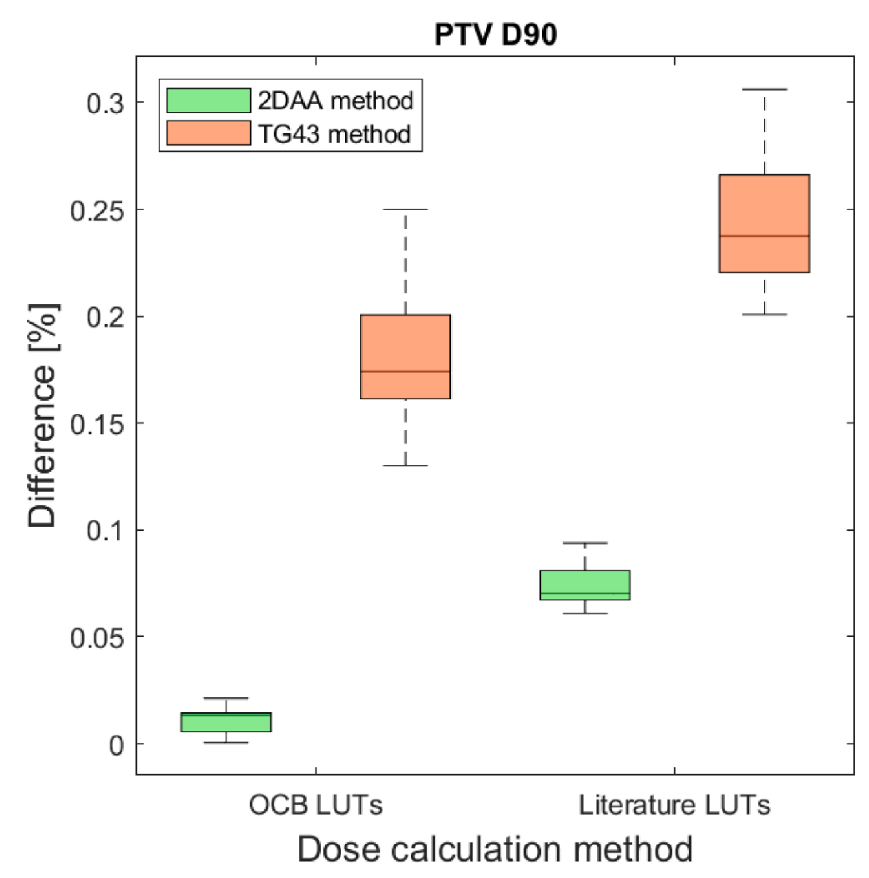

Fig. 3. Differences in the calculated PTV D90\% DVH index comparing clinical patient plans exported from OCB with the plans recalculated in MaxiCalc for 10 plans. A positive difference represents OCB calculating a higher DVH index. The horizontal line in the box represents the mean value, the coloured box represents the 25 th and 75 th percentiles, and the whiskers represent the range.

\section{Discussion}

\subsection{Dose calculation}

\subsubsection{Point dose module}

Table 1 shows an excellent agreement between OCB and MaxiCalc when using the same LUTs used by the TPS with mean and maximum differences of $0 \%$ and $0.01 \%$ across all validation points. As shown in the
Table 5

Planned and measured DVH indices as calculated in MaxiCalc for a clinically delivered plan in which source tracking was performed. The measured dwell positions were tracked and then transformed into the TPS measurement space.

\begin{tabular}{lcc}
\hline DVH Index & Planned DVH indices & Measured DVH indices \\
\hline Prostate D90 [\%] & 102.8 & 106.9 \\
Prostate V100 [\%] & 92.0 & 95.8 \\
Prostate V150 [\%] & 17.5 & 21.5 \\
Prostate V200 [\%] & 6.9 & 8.3 \\
Urethra D10 [\%] & 108.1 & 116.8 \\
Rectum D2cc [\%] & 65.6 & 65.2 \\
Bladder V84 [cm $\left.{ }^{3}\right]$ & 3.1 & 3.7 \\
\hline
\end{tabular}

Supplementary Data 1, differences are attributable to the OCB data being truncated to 4 decimal places. When using the literature data, a good agreement is still seen with mean and maximum differences of $0.07 \%$ and $0.30 \%$. This indicates that the point dose module of MaxiCalc is correctly calculating dose.

The TG43U1 calculations in MaxiCalc and OCB can be evaluated by comparing them to the primary Monte Carlo data [20] they are derived from, where better than $2 \%$ agreement is required. [17] Both MaxiCalc and OCB differ similarly from the primary data, with differences under $2 \%$ as shown in Table 6 . These differences arise from a combination of coarse sampling of the LUTs, and mathematical fits to the primary data that is used to generate the LUTs.

Table 1 shows that, when the goal is to produce a dose calculation engine that replicates that of the clinical TPS, using LUTs with the same reference values (i.e., same sets of angles and distances) gives the most accurate results. While unsurprising, it highlights a potential cause of difference that could easily be overlooked, and which might then contribute to inaccurate estimates of dosimetric differences.

\subsubsection{Volumetric dose module}

Table 2 and Table 3 show that the two different calculation methods and LUTs lead to similar levels of agreement with the TPS. When calculations are performed in MaxiCalc using the same methods and LUTs as the TPS uses, MaxiCalc agrees more closely with OCB than when different methods or LUTs are used. This is expected and agrees with the results from Section 4.1.1.

While Table 2 shows that overall agreement across the entire dose grid is high, Table 3 shows that the differences occur predominantly in the higher dose and dose-gradient regions near the dwells (0.5-15 mm). This is attributable to minor differences in the interpolation methods employed. In the $50 \%$ to $200 \%$ range, the 2DAA approach agrees better than TG43 due to using the same interpolation as the TPS - linearly interpolating through a coarse along-and-away table ( $1 \mathrm{~mm}$ resolution). In contrast, TG43 approximates the inverse square law (ISL) fall off with the geometry function, whose curvature generates slightly lower values at interpolated voxels. The overestimation of interpolated doses is more pronounced in high gradients where the ISL curvature is strongest, resulting in slightly higher DVH indices for the 2DAA method,

Table 6

Local dose differences relative to the published primary Monte Carlo data from Daskalov et al [20] for the 27 dose rate points used to validate OCB v4.6 for three dose calculation engines. MaxiCalc results are shown for two sets of LUTs: one that has interpolation and extrapolation matching $\mathrm{OCB}$, and one verbatim from the literature. Differences are shown in terms of the mean and maximum unsigned (i.e., absolute) dose difference, as well as the number of points that have a difference $>0.5 \%$ and $1 \%$.

\begin{tabular}{lllll}
\hline Dose calculation engine & $\begin{array}{l}\text { Mean (abs) } \\
{[\%]}\end{array}$ & $\begin{array}{l}\text { Max (abs) } \\
{[\%]}\end{array}$ & $\begin{array}{l}\#> \\
0.5 \%\end{array}$ & $\begin{array}{l}\#> \\
1 \%\end{array}$ \\
\hline OCB v4.6 & 0.52 & 2.03 & $13 / 27$ & $3 / 27$ \\
MaxiCalc (OCB LUTs) & 0.52 & 2.01 & $13 / 27$ & $3 / 27$ \\
MaxiCalc (published & 0.50 & 2.01 & $11 / 27$ & $3 / 27$ \\
$\quad$ LUTs) & & & \\
\hline
\end{tabular}


particularly for high-dose metrics and near-PTV volumes.

The faster 2DAA calculations $(<0.08 \mathrm{~s}$ per dwell) allow for MaxiCalc to be used in conjunction with an online source tracking system. Calculation times in the order of the shortest dwell times enable dose contributions to be computed in real time.

\subsection{DVH calculations}

\subsubsection{Validation of $D V H$ index calculations}

The implementation of the DVH indices calculation in MaxiCalc is shown by Table 4 to be valid. Good overall good agreement is seen between OCB and MaxiCalc for all DVH indices of interest. Previous publications have shown that different TPSs calculate different DVH indices for the same plan. [23] Differences in volume calculations and surface definitions also play a part, as seen in Figure 3 in the work by Kirisits et al. [23] This effect is exacerbated when a structure is in a high dose gradient region, which is especially relevant in brachytherapy. A dose verification tool should, when aiming to match the clinical TPS, employ the same dose calculation technique (e.g., calculation method and reference data) to minimise spurious influences.

\subsubsection{DVH index calculations from MaxiCalc dose grids}

DVH indices from the volumetric doses calculated in MaxiCalc for the 10 clinical plans agree mostly within $0.05 \%$ for the 2DAA method or $0.3 \%$ for the TG43 method when compared to the dose grid calculated in and exported from the TPS. This is the case for all DVH indices except for the PTV V200\% where larger differences are seen, where median differences are around $+5 \%$ for the 2DAA method and $+10 \%$ for the TG43 method (i.e., MaxiCalc calculating a lower dose). The PTV V200\% is much more sensitive for two reasons. The volume that receives $200 \%$ of the prescription dose is smaller, and so any differences in structure segmentation at the periphery of the structure have a larger effect on the overall volume due to the larger surface area to volume ratio. It is also in a very high dose gradient region - in the order of $<3 \mathrm{~mm}$ from clinical dwell positions - causing any small difference in calculation of distance or angle to have a large effect on the calculated dose. The overall agreement shows that, aside from the high dose gradient region very near the source, MaxiCalc generates plans that are clinically equivalent to OCB, as seen by the DVH indices agreeing. This justifies the use of MaxiCalc as a tool to calculate indices from measured dwell positions to assess the impact of any deviations in treatment delivery.

When using MaxiCalc as a tool to calculate delivered doses and DVH indices from measured dwell positions, one could also use MaxiCalc to re-calculate the planned dose and indices. This way influences from calculation algorithms are eliminated, and any differences seen can be interpreted in terms of changes from the planned dose due to dwell position variations.

\subsection{Dose calculations from tracked dwell positions}

As MaxiCalc can calculate 3D dose from arbitrary dwell positions, i. e., not constrained to lie on the catheter paths defined in the planning images, the dose and DVH indices can be calculated for a clinically delivered treatment when the source is tracked throughout delivery. This allows for clinically relevant feedback to be provided to clinical staff to either confirm dosimetrically correct treatment delivery, or for any observed deviations to be understood.

The calculations are fast enough to provide real-time feedback during treatment and could be used in several ways. Source-tracking is performed in real-time, and if a discrepancy is detected the treatment can be interrupted or paused while a dosimetric evaluation is conducted to aid the decision to proceed or adapt. This could involve a comparison of the dose contributions of those dwells already delivered to the corresponding subset of the plan. Alternatively, dosimetric analysis could be provided by combining calculated dose from delivered and measured dwells with the planned dose from remaining dwells. This could be continuously updated as more dwell positions are tracked. This approach allows the most up-to-date DVH data to be shown while still providing data that represents dose across the entire contoured volumes and relative to the plan objectives.

When assessing any observed differences from the plan, care needs to be taken to understand the effect of measurement uncertainties. Both source position and time measurements have associated uncertainties which will, in turn, affect the measured 3D dose and DVH indices.

There are two important considerations:

(i) where a dwell measurement differs from the planned position or time by less than the measurement uncertainty, the planned value should be used for the subsequent dose calculation. This is tantamount to accepting the null hypothesis, i.e., no evidence that the delivered dwell is not equal to that planned.

(ii) for dwells that are accepted as different from the plan, measurement uncertainties translate to uncertainties in the calculation of dose metrics. The dependencies are not simple, but it is clear that the clinically accepted range for DVH metrics from source tracking are likely to be wider than those applied to the original planning goals.

The detailed interplay between uncertainty contributions is nontrivial. For example, relative measurements will have smaller uncertainties than absolute position with respect to anatomy due to the requirement for coordinate space registrations. This will have different implications for how to implement a tool such as MaxiCalc to provide the best clinical feedback. Detailed assessment of the use of such a tool and the implications is currently being undertaken.

\section{Conclusions}

MaxiCalc is a novel tool that enables dosimetric treatment verification and evaluation to be performed when used in conjunction with any source tracking-based treatment verification system, capable of measuring source positions relative to patient anatomy. Good agreement with a clinical TPS when calculating both point and volumetric doses has been shown. MaxiCalc can calculate DVH indices independent of any other software, enabling a standalone device to calculate and report delivered brachytherapy doses to the clinical treatment staff. It is fast enough to be used in real-time where it could be used for evaluation of a partial treatment delivery that has been paused or interrupted.

When a tool such as MaxiCalc is used to evaluate the dosimetric impact of any deviations from the planned treatment delivery, care should be taken to use the same dose calculation methods and reference data (e.g., LUTs) for both the planned and measured dwells to minimise any differences inherent in the calculation approach. This should be done by either ensuring that the custom dose calculation engine uses the same calculation method and reference data used by the clinical TPS, or by using the custom dose calculation engine for both the planned and measured dwells to determine dosimetric differences. Ideally, the same algorithm should be used for calculating DVH indices from these doses.

The use of MaxiCalc also enables the possibility of calculating patient-specific and plan-specific error thresholds, as well as adaptive planning for the current pre-treatment imaging protocol at AHRO. The determination of patient-specific error detection thresholds allows for action limits to be set that are unique to the treatment verification system, clinical procedure, and patient based on a level of change that would cause unacceptable dosimetry for that individual patient.

MaxiCalc offers a method to perform 3D dose and DVH index analysis for any source tracking-based treatment verification system, regardless of the technology used. This work details a method to test and validate an independent dose analysis tool for the interpretation of source-tracking data in terms of clinical significance. 


\section{Acknowledgements}

The authors acknowledge that the work was performed on the unceded lands of the Wurundjeri people and Bunurong people of the Kulin Nation. We acknowledge their connects to the land and waters on which we live and practice our science and acknowledge their Elders past and present. The authors would like to thank Dr Emily Poon and the staff at the McGill University Health Centre (MUHC) for allowing us to adapt the dose structure exporting code from BrachyGUI. MH would like to acknowledge that this research is supported by an Australian Government Research Training Program (RTP) Scholarship.

\section{Appendix A. Supplementary data}

Supplementary data to this article can be found online at https://doi. org/10.1016/j.ejmp.2021.12.008.

\section{References}

[1] Smith R, McDermott L, Franich R. Treatment dosimetry for HDR brachytherapy with an EPID. Radiother Oncol 2009;92:S140-1. https://doi.org/10.1016/s01678140(12)72954-7.

[2] Smith RL, Taylor ML, McDermott LN, Haworth A, Millar JL, Franich RD. Source position verification and dosimetry in HDR brachytherapy using an EPID. Med Phys 2013;40(11):111706. https://doi.org/10.1118/1.4823758.

[3] Smith RL, Hanlon M, Panettieri V, Millar JL, Matheson B, Haworth A, et al. An integrated system for clinical treatment verification of HDR prostate brachytherapy combining source tracking with pretreatment imaging. Brachytherapy $2018 ; 17(1)$ : 111-21. https://doi.org/10.1016/j.brachy.2017.08.004.

[4] Johansen JG, Kertzscher G, Jørgensen EB, Rylander S, Bentzen L, Hokland SB, et al. Dwell time verification in brachytherapy based on time resolved in vivo dosimetry. Phys Med 2019;60:156-61. https://doi.org/10.1016/j.ejmp.2019.03.031.

[5] Jørgensen EB, Kertzscher G, Buus S, Bentzen L, Hokland SB, Rylander S, et al. Accuracy of an in vivo dosimetry-based source tracking method for afterloading brachytherapy - A phantom study. Med Phys 2021;48(5):2614-23. https://doi. org/10.1002/mp.v48.510.1002/mp.14812.

[6] Linares Rosales HM, Johansen JG, Kertzscher G, Tanderup K, Beaulieu L, Beddar S. 3D source tracking and error detection in HDR using two independent scintillator dosimetry systems. Med Phys 2021;48(5):2095-107. https://doi.org/10.1002/mp. v48.510.1002/mp.14607.

[7] Mason J, Henry A, Bownes P. Error detection thresholds for routine real time in vivo dosimetry in HDR prostate brachytherapy. Radiother Oncol 2020;149:38-43. https://doi.org/10.1016/j.radonc.2020.04.058.

[8] Romanyukha A, Carrara M, Mazzeo D, Tenconi C, Al-Salmani T, Poder J, et al. An innovative gynecological HDR brachytherapy applicator system for treatment delivery and real-time verification. Phys Med 2019;59:151-7. https://doi.org/ 10.1016/j.ejmp.2019.03.001.
[9] Nose T, Chatani M, Otani Y, Teshima T, Kumita S. Real-Time Verification of a HighDose-Rate Iridium 192 Source Position Using a Modified C-Arm Fluoroscope. Int J Radiat Oncol Biol Phys 2017;97(4):858-65. https://doi.org/10.1016/j. ijrobp.2016.12.005.

[10] Watanabe Y, Muraishi H, Takei H, Hara H, Terazaki T, Shuto N, et al. Automated source tracking with a pinhole imaging system during high-dose-rate brachytherapy treatment. Phys Med Biol 2018;63(14):145002. https://doi.org/ 10.1088/1361-6560/aacdc9.

[11] Safavi-Naeini M, Han Z, Alnaghy S, Cutajar D, Petasecca M, Lerch MLF, et al. BrachyView, a novel in-body imaging system for HDR prostate brachytherapy: Experimental evaluation. Med Phys 2015;42(12):7098-107. https://doi.org/ 10.1118/1.4935866.

[12] Beld E, Moerland MA, Zijlstra F, Viergever MA, Lagendijk JJW, Seevinck PR. MRbased source localization for MR-guided HDR brachytherapy. Phys Med Biol 2018; 63(8):085002. https://doi.org/10.1088/1361-6560/aab50b.

[13] Fonseca GP, Podesta M, Bellezzo M, Van den Bosch MR, Lutgens L, Vanneste BGL, et al. Online pretreatment verification of high-dose rate brachytherapy using an imaging panel. Phys Med Biol 2017;62(13):5440-61. https://doi.org/10.1088/ 1361-6560/aa7028.

[14] Poder J, Cutajar D, Guatelli S, Petasecca M, Howie A, Bucci J, et al. A Monte Carlo study on the feasibility of real-time in vivo source tracking during ultrasound based HDR prostate brachytherapy treatments. Phys Med 2019;59:30-6. https://doi.org/ 10.1016/j.ejmp.2019.02.012.

[15] Johansen JG, Rylander S, Buus S, Bentzen L, Hokland SB, Søndergaard CS, et al. Time-resolved in vivo dosimetry for source tracking in brachytherapy. Brachytherapy 2018;17:122-32. https://doi.org/10.1016/j.brachy.2017.08.009.

[16] Poder J, Cutajar D, Guatelli S, Petasecca M, Howie A, Bucci J, et al. HDR brachytherapy in vivo source position verification using a 2D diode array: A Monte Carlo study. J Appl Clin Med Phys 2018;19(4):163-72. https://doi.org/10.1002/ acm2.2018.19.issue-410.1002/acm2.12360.

[17] Rivard MJ, Coursey BM, DeWerd LA, Hanson WF, Huq MS, Ibbot GS, et al. Update of AAPM Task Group No. 43 Report: A revised AAPM protocol for brachytherapy dose calculations. Med Phys 2004;3:633-74. 10.1118/1.1646040.

[18] Fonseca GP, Johansen JG, Smith RL, Beaulieu L, Beddar S, Kertzscher G, et al. In vivo dosimetry in brachytherapy: Requirements and future directions for research, development, and clinical practice. Phys Imaging Radiat Oncol 2020;16:1-11. https://doi.org/10.1016/j.phro.2020.09.002.

[19] Poder J, Carrara M, Howie A, Cutajar D, Bucci J, Rosenfeld A. Derivation of in vivo source tracking error thresholds for TRUS-based HDR prostate brachytherapy through simulation of source positioning errors. Brachytherapy 2019;18:711-9. https://doi.org/10.1016/j.brachy.2019.05.001.

[20] Daskalov GM, Löffler E, Williamson JF. Monte Carlo-aided dosimetry of a new high dose-rate brachytherapy source. Med Phys 1998;25(11):2200-8. https://doi.org/ $10.1118 / 1.598418$

[21] Elekta AB. Oncentra ${ }^{\circledR}$ Brachy v4.6 Physics and Algorithms 2018.

[22] Poon E, Le Y, Williamson JF, Verhaegen F. An adjunct to an accelerated Monte Carlo photon transport code for patient-specific brachytherapy dose calculations and analysis. J Phys Conf Ser 2008;102:012018. https://doi.org/10.1088/17426596/102/1/012018.

[23] Kirisits C, Siebert F-A, Baltas D, De Brabandere M, Hellebust TP, Berger D, et al. Accuracy of volume and DVH parameters determined with different brachytherapy treatment planning systems. Radiother Oncol 2007;84(3):290-7. https://doi.org/ 10.1016/j.radonc.2007.06.010. 